\title{
The BEM for buckling analysis of viscoelastic plates modelled with fractional derivatives
}

\author{
J. T. Katsikadelis \& N. G. Babouskos \\ School of Civil Engineering, \\ National Technical University of Athens, Greece
}

\begin{abstract}
In this paper the buckling of viscoelastic plates is studied. The constitutive equations of the viscoelastic material are expressed in differential form using fractional derivatives. The proposed analysis is illustrated with the fractional Kelvin-Voigt and fractional Standard solid models. Plates of arbitrary shape with any type of boundary conditions under interior and edge conservative membrane loads are considered. The principle of the analog equation is applied to convert the original equation into a plate equation (biharmonic) under a fictitious load. Subsequent application of the BEM enables the spatial discretization resulting thus an initial value problem for the values of the fictitious load, which is a system of linear Fractional Differential Equations (FDEs) with respect to time. Using a property of the Mittag-Leffler function a dynamic criterion is established and the eigenvalue problem for the evolution equations is converted into an eigenvalue problem of linear algebra, which permits the evaluation of the buckling loads of the viscoelastic plate. Several plate problems are studied and interesting conclusions on the effect of viscoelasticity on bucking of thin plates are drawn.

Keywords: thin plates, viscoelastic, fractional derivative models, buckling, boundary element method, analog equation method.
\end{abstract}

\section{Introduction}

The buckling of viscoelastic structures modelled with integral or integer order differential constitutive equations has been investigated by several authors [1-3]. There are papers dealing with the stability of viscoelastic beams [4-7] and viscoelastic plates under conservative loads [8-10] using such models. In the last 
years many researchers have shown that the response of real viscoelastic structures is described accurately using fractional derivatives with much less parameters than the classical models. However their use leads to partial fractional differential equations, whose mathematical treatment is very difficult. Therefore the literature is limited on viscoelastic structures modelled with fractional derivatives [11-13]. Recently, Katsikadelis developed a numerical method to solve ordinary fractional differential equations [14] that was extended to partial ones. This paved the way to analyse viscoelastic structures described with fractional derivative models. Using this technique Katsikadelis and Babouskos [15] studied recently the postbuckling response of viscoelastic plates modelled with fractional derivatives.

In this paper the linear buckling of viscoelastic plates modelled with generalized fractional differential constitutive equations is investigated. The plate has an arbitrary shape and is subjected to any type of boundary conditions under interior and edge conservative membrane loads. The governing equation and the boundary conditions are first written in terms of stress resultants. Then application of the differential operator of the viscoelastic constitutive equations $[1,7]$ yields an evolution partial differential equation in terms of the transverse displacement. The proposed analysis is illustrated with two fractional viscoelastic models, namely the fractional Kelvin-Voigt model and the fractional Standard solid model. The principle of the analog equation [16] is applied to convert the original equation into a plate equation (biharmonic) under a time dependent fictitious load, unknown in the first instance. Then application of the BEM yields the semi-discrete evolution equations, which is a system of linear ordinary FDEs. Assuming the Mittag-Leffler function [17] as the solution of homogeneous system, we obtain an eigenvalue problem of linear algebra, which permits the evaluation of the buckling loads. Several plate problems are examined and interesting conclusions on the effect of viscoelasticity on bucking of thin plates are drawn.

\section{Governing equations}

We consider a thin elastic plate of uniform thickness $h$ occupying the two dimensional multiply connected domain $\Omega$ of the $x y$ plane with boundary $\Gamma \cup_{i=0}^{K} \Gamma_{i}$ (Fig. 1). The non intersecting curves $\Gamma_{i}(i=0,1,2, \ldots, K)$ may be piece-wise smooth. There are $N_{c}$ corner points along the boundary. The boundary may be simply supported, clamped, free or elastically supported with transverse stiffness $k_{T}(\mathbf{x})$ and rotational stiffness $k_{R}(\mathbf{x}), \mathbf{x}:(x, y) \in \Gamma$. The plate is subjected to inplane loads $p_{x}, p_{y}$ inside $\Omega$ as well as edge inplane forces $N_{n}^{*}, N_{t}^{*}$.

Applying the principle of virtual work, we obtain

$$
\begin{aligned}
M_{x, x x}+ & 2 M_{x y, x y}+M_{y}, y y+N_{x} w, x x \\
& +2 N_{x y} w_{, x y}+N_{y} w_{, y y}-p_{x} w_{, x}-p_{y} w_{, y}=0
\end{aligned}
$$




$$
\begin{gathered}
V_{n}+N_{n}^{*} w, n+N_{t}^{*} w,{ }_{t}+k_{T} w=0 \text { or } w=0 \text { on } \Gamma \\
M_{n}-k_{R} w,_{n}=0 \text { or } w,_{n}=0 \quad \text { on } \Gamma \\
k_{T}^{(k)} w^{(k)}-\llbracket M_{n t} \rrbracket_{k}=0 \text { or } w^{(k)}=0 \quad \text { at corner point k }
\end{gathered}
$$

where $w$ is the transverse deflection of the plate and $M_{x}, M_{y}, M_{x y}$ are the bending and twisting moments of the plate; $V_{n}$ and $M_{n}$ are the equivalent shear force and the normal bending moment along the boundary and $\llbracket M_{n t} \rrbracket_{k}=M_{n t}^{+}-M_{n t}^{-}$is the discontinuity jump of the twisting moment at the corner point $k . N_{x}, N_{y}, N_{x y}$ are the stress resultants due to the membrane loading obtained from the solution of plane stress problem and are assumed to be time independent, that is they are obtained from the elastic solution. In this investigation, the plane stress problem is solved using the conventional BEM [18].

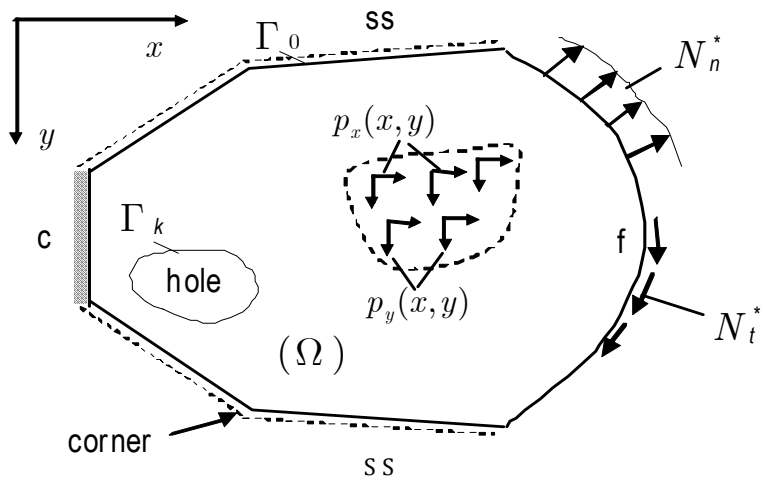

Figure 1: Plate geometry and supports ( $\mathrm{c}=$ clamped, $\mathrm{ss}=$ simply supported, $f=$ free).

For the elastic material the stress resultants are written as

$$
\begin{gathered}
M_{x}=-D\left(w,_{x x}+\nu w, y y\right), M_{y}=-D\left(\nu w,_{x x}+w, y y\right) \\
M_{x y}=-D(1-\nu) w, x y \\
V_{n}=V w, \quad M_{n}=M w, \quad M_{n t}=T w
\end{gathered}
$$

where $D=E h^{3} /\left(1-\nu^{2}\right)$ is the flexural rigidity of the plate. The operators $V, M$ and $T$ are given as [19]

$$
\begin{gathered}
V=-D\left[\frac{\partial}{\partial n} \nabla^{2}+(1-\nu) \frac{\partial}{\partial s}\left(\frac{\partial^{2}}{\partial s \partial n}-\kappa \frac{\partial}{\partial s}\right)\right] \\
M=-D\left[\nabla^{2}-(1-\nu)\left(\frac{\partial^{2}}{\partial s^{2}}+\kappa \frac{\partial}{\partial n}\right)\right], T=D(1-\nu)\left(\frac{\partial^{2}}{\partial s \partial n}-\kappa \frac{\partial}{\partial s}\right)(5 \mathrm{~b}, \mathrm{c})
\end{gathered}
$$


The constitutive equations of the viscoelastic material are written in differential form $[1,20]$ and can be generalized by replacing the integer order derivatives with fractional derivatives [11, 12]. Thus for the plate bending problem the viscoelastic constitutive equations can be written as

$$
\begin{gathered}
\mathbf{P} M_{x}=-D \mathbf{Q}(w, x x+\nu w, y y) \\
\mathbf{P} M_{y}=-D \mathbf{Q}(\nu w, x x+w, y y) \\
\mathbf{P} M_{x y}=-D(1-\nu) \mathbf{Q} w, x y
\end{gathered}
$$

where $\mathbf{P}, \mathbf{Q}$ are fractional differential operators with respect to time defined as

$$
\mathbf{P}=\sum_{k=0}^{l} p_{k} D_{c}^{\alpha_{k}}, \mathbf{Q}=\sum_{k=0}^{m} q_{k} D_{c}^{\alpha_{k}}
$$

in which $D_{c}^{a_{k}}$ is the Caputo fractional derivative of order $\alpha_{k}$, with $\alpha_{0}=0$, and $p_{k}, q_{k}$ are the viscoelastic parameters, which should satisfy certain conditions resulting from second law of thermodynamics [11]. Note that for $l=m=0$ it is $\mathbf{P}=\mathbf{Q}=1$ and eqns (6) give the constitutive equations (3) of the elastic material. Similarly the equivalent shear force, the normal bending moment and the twisting moment along the boundary can be written as

$$
\mathbf{P} V_{n}=\mathbf{Q} V w, \mathbf{P} M_{n}=\mathbf{Q} M w, \mathbf{P} M_{n t}=\mathbf{Q} T w \quad(8 \mathrm{a}, \mathrm{b}, \mathrm{c})
$$

Applying operator $\mathbf{P}$ on eqns (1) and (2) we obtain the equations and the boundary conditions of the plate bending problem in terms of the transverse deflection $w[1,7]$. Thus we obtain

$$
\begin{gathered}
\mathbf{P} M_{x, x x}+2 \mathbf{P} M_{x y, x y}+\mathbf{P} M_{y, y y}+N_{x} \mathbf{P} w,_{x x} \\
+2 N_{x y} \mathbf{P} w_{, x y}+N_{y} \mathbf{P} w_{y y}-p_{x} \mathbf{P} w_{, x}-p_{y} \mathbf{P} w_{, y}=0 \\
\mathbf{P} V_{n}+N_{n}^{*} \mathbf{P} w_{n}+N_{t}^{*} \mathbf{P} w_{, t}+k_{T} \mathbf{P} w=0 \text { or } \quad w=0 \quad \text { on } \Gamma \\
\mathbf{P} M_{n}+k_{R} \mathbf{P} w_{n}=0 \text { or } w,_{n}=0 \quad \text { on } \Gamma \\
k_{T}^{(k)} \mathbf{P} w^{(k)}-\llbracket \mathbf{P} M_{n t} \rrbracket_{k}=0 \text { or } w^{(k)}=0 \quad \text { at corner point } \mathrm{k}
\end{gathered}
$$

which by virtue of eqn (6) and (8) become

$$
\begin{gathered}
\mathbf{Q} D \nabla^{4} w-N_{x} \mathbf{P} w_{, x x}-2 N_{x y} \mathbf{P} w_{, x y} \quad \text { in } \Omega \\
-N_{y} \mathbf{P} w_{y y}+p_{x} \mathbf{P} w_{, x}+p_{y} \mathbf{P} w_{y}=0 \\
\mathbf{Q} V w+N_{n}^{*} \mathbf{P} w,_{n}+N_{t}^{*} \mathbf{P} w_{, t}+k_{T} \mathbf{P} w=0 \text { or } w=0 \quad \text { on } \Gamma \\
\mathbf{Q} M w+k_{R} \mathbf{P} w,_{n}=0 \text { or } w_{, n}=0 \quad \text { on } \Gamma \\
k_{T}^{(k)} \mathbf{P} w^{(k)}-\llbracket \mathbf{Q} T w \rrbracket_{k}=0 \text { or } w^{(k)}=0 \text { at corner point } \mathrm{k}
\end{gathered}
$$

The above equations are fractional differential equations with respect to time. The number of the initial conditions that are needed for the solution of eqn (11)(12) depends on the order of the viscoelastic constitutive equation. Thus in the general case the initial conditions can be written as

$$
\frac{d^{k} w(\mathbf{x}, 0)}{d t^{k}}=g_{k}(\mathbf{x}),(k=0,1,2 . ., n)
$$

where $g_{k}(\mathbf{x})$ is a known function and $n \in \mathbb{N}$ with $n-1<\alpha_{k}<n$. 
For the fractional Kelvin-Voigt model (Fig 2a) it is $l=0, m=1$ and $p_{0}=1, q_{0}=1$ with $q_{1}=\eta$ being the viscous parameter. Thus eqn (11) becomes

$$
\begin{aligned}
D \nabla^{4} w+ & \eta D D_{c}^{\alpha} \nabla^{4} w-N_{x} w,_{x x} \\
& -2 N_{x y} w,_{x y}-N_{y} w,_{y y}+p_{x} w,_{x}+p_{y} w,_{y}=0
\end{aligned}
$$

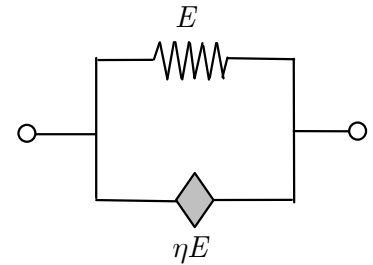

(a)

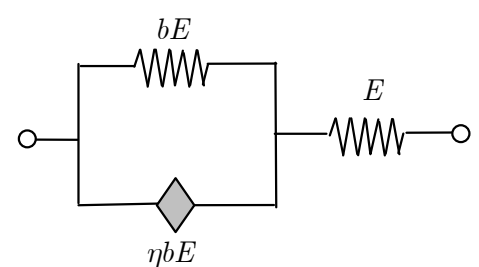

(b)

Figure 2: (a) The fractional Kelvin-Voigt model and (b) the fractional Standard solid model in uniaxial representation.

For the fractional Standard solid model (Fig. 2b) it is $l=m=1$ and $p_{0}=1$, $p_{1}=\eta b /(1+b), \quad q_{0}=b /(1+b), \quad q_{1}=\eta b /(1+b) \quad[21]$ where $b, \eta$ are parameters of the viscoelastic material. Thus eqn (11) becomes

$$
\begin{aligned}
& q_{0} D \nabla^{4} w+q_{1} D D_{c}^{\alpha} \nabla^{4} w-N_{x}\left(w, x x+p_{1} D_{c}^{\alpha} w, x x\right) \\
& -2 N_{x y}(w, x y \\
& \left.\quad+p_{1} D_{c}^{\alpha} w, x y\right)-N_{y}\left(w, y y+p_{1} D_{c}^{\alpha} w, y y\right) \\
& \quad+p_{x}\left(w, x_{x}+p_{1} D_{c}^{\alpha} w, x\right)+p_{y}\left(w, y+p_{1} D_{c}^{\alpha} w, y\right)=0
\end{aligned}
$$

Equations (14) and (15) are fractional differential equations of order $0<\alpha \leq 1$ with respect to time, therefore only one initial condition, namely the initial displacement, is required. Since we study the stability of the plate, we suppose that the plate is initially undeformed. Thus the initial condition is

$$
w(\mathbf{x}, 0)=0
$$

In this investigation the time dependent terms in the boundary conditions of the viscoelastic plate are neglected. It can be shown that when the plate is initially undeformed and for $k_{T}=k_{R}=0$ the time dependent terms do not appear in the boundary conditions. The influence of these terms will be the subject of a forthcoming paper. Thus, the boundary conditions for both viscoelastic models are given by eqn (12) with $\mathbf{P}=\mathbf{Q}=1$.

\section{The solution procedure}

The initial boundary value problem (11)-(12) is solved using the AEM. The analog equation for the problem at hand is 
40 Boundary Elements and Other Mesh Reduction Methods XXXIII

$$
\nabla^{4} w=b(\mathbf{x}, t), \quad \mathbf{x}=\{x, y\} \in \Omega
$$

where $b(\mathbf{x}, t)$ represents a fictitious load, unknown in the first instance. Eqn (17) under the boundary conditions (12) is solved using the BEM as presented in [15]. This yields

$$
w, p q r(\mathbf{x}, t)=\sum_{k=1}^{M} b_{k}(t) W_{k}, p q r(\mathbf{x}), p, q, r=0, x, y \quad \mathbf{x} \in \Omega
$$

where $W_{k}, p q r$ are known functions and $b_{k}(t)$ the values of the fictitious load at the $M$ collocation points inside $\Omega$. Note that the above notation implies $w, 000=w, w, 0 y 0=w, y$, etc. Applying eqn (18) for the $M$ internal nodal points we obtain

$$
\mathbf{w}, p q r=\mathbf{W}, p q r \mathbf{b}
$$

Further, collocating the governing equations at the $M$ internal nodal points and using eqn (19) to express the involved derivatives in terms of $\mathbf{b}$ we obtain:

(i) For the elastic plate

$$
(\mathbf{K}-\mu \mathbf{F}) \mathbf{b}=0 \text { in } \Omega
$$

where $\mu$ is the buckling parameter and $\mathbf{K}, \mathbf{F}$ are $M \times M$ known matrices defined as

$$
\begin{gathered}
\mathbf{K}=D \mathbf{I} \\
\mathbf{F}=\mathbf{N}_{\mathbf{x}} \mathbf{W}_{, \mathbf{x x}}+2 \mathbf{N}_{\mathbf{x y}} W,{ }_{, x y}+\mathbf{N}_{\mathbf{y}} \mathbf{W}, y y-\mathbf{p}_{\mathbf{x}} W,{ }_{, x}-\mathbf{p}_{\mathbf{y}} W, \mathbf{y}
\end{gathered}
$$

with $\mathbf{I}$ being the identity matrix and $\mathbf{N}_{\mathbf{x}}, \mathbf{N}_{\mathbf{x y}}, \mathbf{N}_{\mathbf{y}}, \mathbf{p}_{\mathbf{x}}, \mathbf{p}_{\mathbf{y}}$ are $M \times M$ diagonal matrices including the values of the membrane forces and membrane loads at the $M$ internal nodal points. It should be noted that in this case the fictitious load $\mathbf{b}$ does not depend on time. Eqn (20) constitutes an eigenvalue problem of linear algebra, which can be solved for the critical values of the load parameter $\mu$.

(ii) For the fractional Kelvin-Voigt plate

$$
\eta \mathbf{K} D_{c}^{\alpha} \mathbf{b}+\mathbf{K}-\mu \mathbf{F}=0
$$

(iii) For the fractional Standard solid plate

$$
\left(q_{1} \mathbf{K}-p_{1} \mu \mathbf{F}\right) D_{c}^{\alpha} \mathbf{b}+q_{0} \mathbf{K}-\mu \mathbf{F}=0
$$

For $\mathbf{b}(0)=0$, eqns (22) and (23) constitute eigenvalue problems for the buckling parameter $\mu$. The stability of the fractional differential equations (22) and (23) can be studied by introducing a dynamic criterion analogous to that for elastic structures. Thus, observing that for Mittag-Leffler function $E_{\alpha, 1}\left(\lambda t^{\alpha}\right)$ holds [17]

$$
D_{c}^{\alpha}\left[E_{\alpha, 1}\left(\lambda t^{\alpha}\right)\right]=\lambda E_{\alpha, 1}\left(\lambda t^{\alpha}\right)
$$

we can assume a solution of eqns (22) and (23) of the form

$$
\mathbf{b}(t)=\boldsymbol{\beta} E_{\alpha, 1}\left(\lambda t^{\alpha}\right)
$$

where $\boldsymbol{\beta}$ is a constant vector, and $\lambda \in \mathbb{C}$ is a parameter. Note that for $\alpha=1$ it is $E_{1,1}=e^{\lambda t}$. Therefore, the parameter $\lambda$ plays here the role of the 
eigenfrequency of free vibrations. Substituting eqn (25) into eqn (22) and taking into account eqn (24) we obtain

$$
(\eta \lambda \mathbf{K}+\mathbf{K}-\mu \mathbf{F}) \boldsymbol{\beta}=0
$$

Similarly eqn (23) becomes

$$
\left[\lambda\left(q_{1} \mathbf{K}-p_{1} \mu \mathbf{F}\right)+q_{0} \mathbf{K}-\mu \mathbf{F}\right] \boldsymbol{\beta}=0
$$

Equations (26) and (27) constitute generalized eigenvalue problems of linear algebra. For a given value of the load parameter $\mu$ the solution of the eigenvalue problems (26) and (27) give the eigenvalue $\lambda$ which in general is a complex number. The stability of the plate is determined by writing the eigenvalue $\lambda$ in polar form $\lambda=\rho e^{i \phi}$ and taking into account the asymptotic response of the Mittag-Leffler function as $t \rightarrow \infty$ [17]:

(a) $E_{\alpha, 1}\left(t^{\alpha} \rho e^{i \phi}\right) \rightarrow 0$ when $|\phi|>\alpha \pi / 2$

(b) $E_{\alpha, 1}\left(t^{\alpha} \rho e^{i \phi}\right)$ remains bounded when $|\phi|=\alpha \pi / 2$

(c) $\left|E_{\alpha, 1}\left(t^{\alpha} \rho e^{i \phi}\right)\right| \rightarrow \infty$ when $|\phi|<\alpha \pi / 2$

Hence, by increasing the load parameter $\mu$ the plate becomes unstable if $|\phi|<\alpha \pi / 2$ (Fig. 3). The threshold of instability is $|\phi|=\alpha \pi / 2$. If the eigenvalue $\lambda$ is a real number the plate becomes unstable when $\lambda>0$.

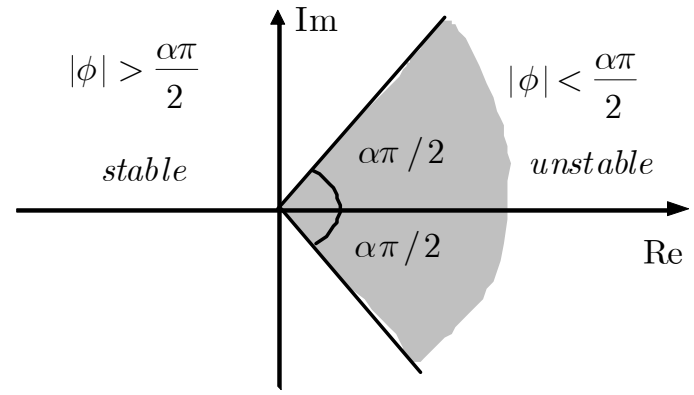

Figure 3: Stability region.

\section{Examples}

\subsection{Example 1}

The simply supported square plate of uniform thickness $h=0.1 \mathrm{~m}$ of Fig. 4 is subjected to a uniform membrane load along the edges with $x=0$ and $x=4$. Due to inplane boundary conditions the produced membrane forces are $N_{x}=-P$ and $N_{x y}=N_{y}=0$. First the elastic plate is studied. The elastic parameters are $E=21 \times 10^{6} \mathrm{kN} / \mathrm{m}^{2}$ and $\nu=0.25$. The results were obtained with $N=200$ boundary elements. 


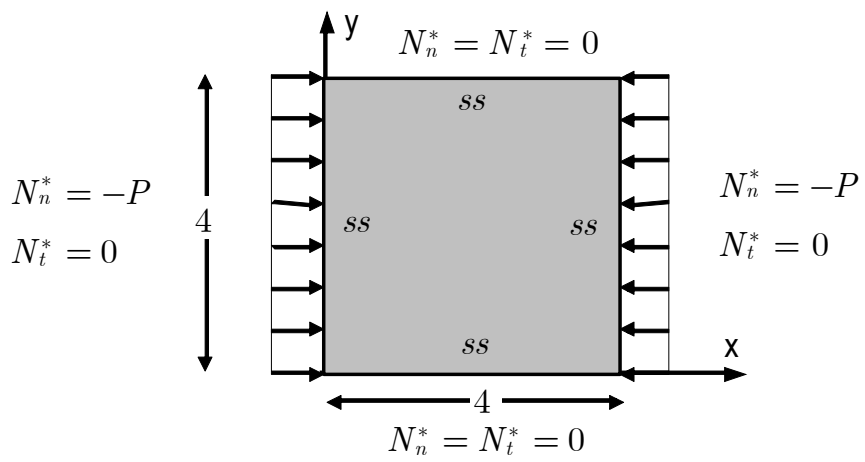

Figure 4: $\quad$ Boundary conditions of plate in example 1.

Table 1 presents the first four buckling loads of the elastic plate for various values of the internal nodal points and the results are compared with the exact ones [22]. Next the response of the Kelvin-Voigt plate is studied. The results were obtained with $M=236$ internal nodal points resulting from 312 linear triangular domain elements. Fig. 5 presents the first four eigenvalues $\lambda$ as the load $P$ increases for $\eta=1$. In this case the eigenvalues are real numbers and

Table 1: Buckling loads of the elastic plate for various values of the internal nodal points.

\begin{tabular}{|c|c|c|c|c|}
\hline \multirow{2}{*}{$M$} & \multicolumn{4}{|c|}{ Buckling loads } \\
\cline { 2 - 5 } & 1 & 2 & 3 & 4 \\
\hline 145 & 4682.40 & 7483.87 & 13789.24 & 19620.16 \\
\hline 236 & 4651.44 & 7371.86 & 13402.42 & 19144.91 \\
\hline 329 & 4636.32 & 7312.76 & 13202.77 & 18901.36 \\
\hline 401 & 4629.79 & 7288.81 & 13118.50 & 18800.78 \\
\hline Exact $[22]$ & 4605.82 & 7196.59 & 12793.93 & 18423.26 \\
\hline
\end{tabular}

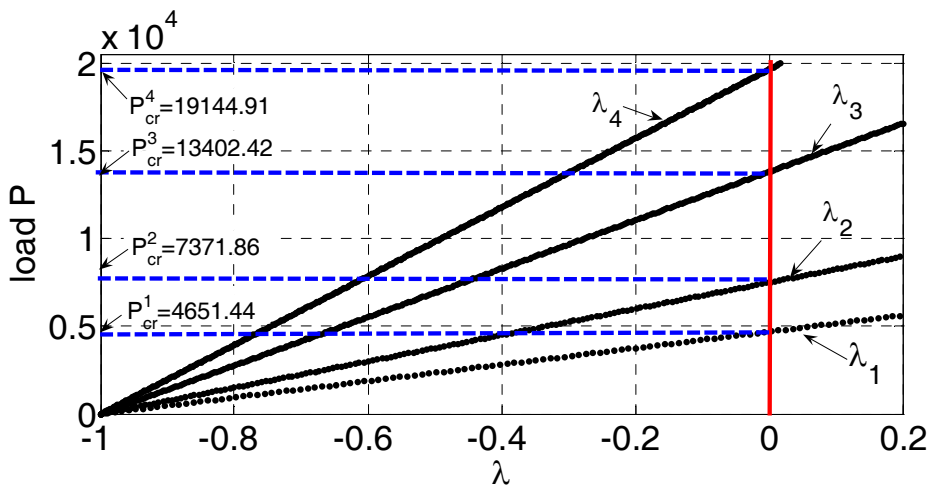

Figure 5: $\quad$ Load-eigenvalue curves for Kelvin-Voigt plate $(\eta=1)$. 
plate becomes unstable when $\lambda>0$. Fig. 6 presents the load $-1^{\text {st }}$ eigenvalue curves for various values of the viscous parameter $\eta$. The critical loads of the Kelvin-Voigt plate are the same with that of the elastic plate. Similar results were obtained in $[1,7]$ who studied the stability of the viscoelastic beams. Finally, the stability results do not depend on the order of the fractional derivative $\alpha$. However, the postbuckling response of the viscoelastic plate is affected by the fractional derivative $\alpha$ as it is shown in [15].

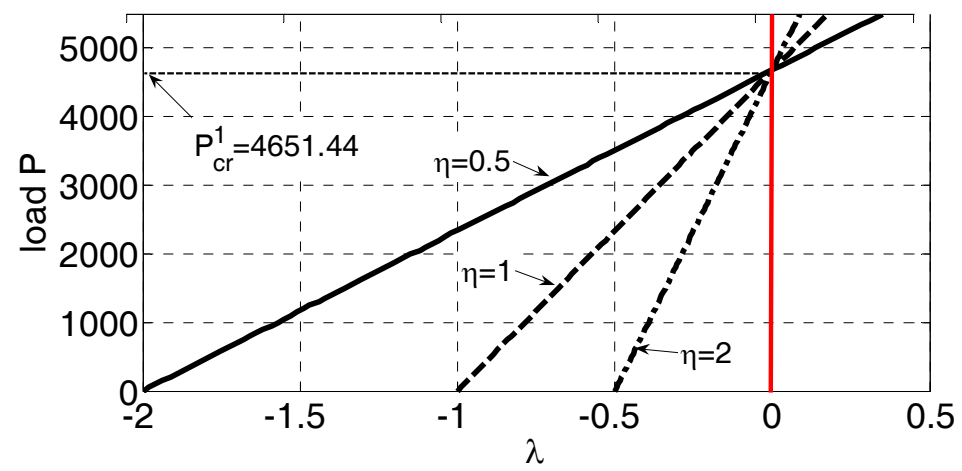

Figure 6: Load- ${ }^{\text {st }}$ eigenvalue curves for various values of $\eta$ for KelvinVoigt plate.

\subsection{Example 2}

The stability of the fractional Standard solid plate of fig. 4 is studied. Fig. 7 presents the $1^{\text {st }}$ eigenvalue as the load $P$ increases for $b=2$ and for various values of $\eta$. The eigenvalues are real numbers and instability occurs when $\lambda>0$. The buckling load does not depend on the parameter $\eta$ or on the order of the fractional derivative $\alpha$. Fig. 8 presents the load-eigenvalue curves for $\eta=1$ and various values of the parameter $b$. It is observed that the buckling load increases as the parameter $b$ increases.

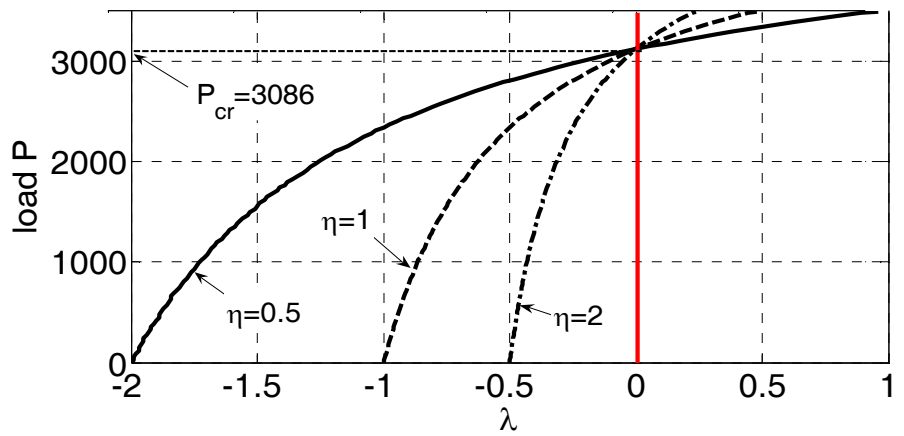

Figure 7: $\quad$ Load- $1^{\text {st }}$ eigenvalue curves for various $\eta(b=2)$. 


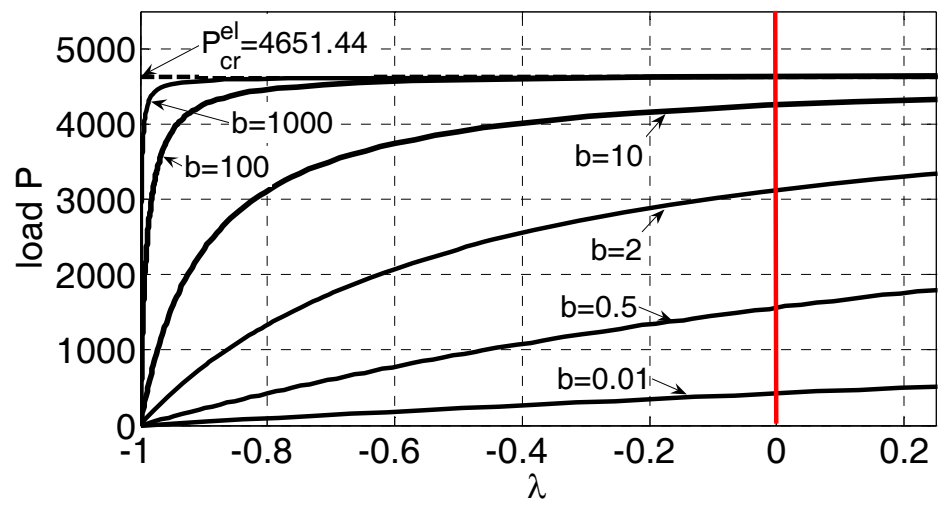

Figure 8: $\quad$ Load- $1^{\text {st }}$ eigenvalue curves for various values of $b(\eta=1)$.

For $b=0$ the buckling load is $P_{c r}=0$. In this case the Standard solid model is transformed to the Maxwell viscoelastic model which is suitable for fluid materials [1]. As the parameter $b \rightarrow \infty$ the buckling load of the viscoelastic plate approaches the buckling load of the elastic plate (see Example 1). In this case the behaviour of the Standard solid model approaches the behaviour of the elastic material. The postbuckling response of the Standard solid model is more complicated and can be studied by integration of the evolution equations $[1,7]$.

\section{Conclusions}

The linear buckling problem of viscoelastic plates is studied. The plate has an arbitrary shape and is subjected to any type of boundary conditions under interior and edge conservative membrane loads. The governing equations are partial fractional differential equations. The AEM is applied to convert the initial boundary value problem of the viscoelastic plate to an initial value problem. Based on a property of the Mittag-Leffler function a dynamic criterion is developed, which converts the initial value problem into an algebraic eigenvalue problem. The latter permits the evaluation of the buckling loads.

It is observed that the stability of the plate does not depend on the order of the fractional derivative $\alpha$ or the viscous parameter $\eta$ of the viscoelastic model, contrary to the postbuckling response which is affected by the viscoelastic model $[7,15]$. For the Standard solid model the parameter $b$ changes significantly the buckling load of the plate. An advantage of the presented procedure is that it avoids the direct time integration of the evolution equations to establish the buckling loads

\section{References}

[1] Flügge, W., Viscoelasticity, Second Revised Edition, Springer-Verlag, Berlin/Heidelberg, 1975. 
[2] Bazant, Z. \& Cedolin, L., Stability of Structures, Elastic, Inelastic, Fracture, and Damage Theories, Oxford University Press, 1991.

[3] Minahen, T. M. \& Knauss, W.G., Creep buckling of viscoelastic structures. Int. J. Solids Structures, 30(8), pp. 1075-1092, 1993.

[4] Chang, W.P., Creep buckling of nonlinear viscoelastic columns. Acta Mech., 60, pp. 199-215, 1986.

[5] Vinogradov, A.M., Buckling of viscoelastic beam columns. AIAA Journal, 25, pp. 479-483, 1987.

[6] Drawshi, M. \& Cederbaum, G., Stability of multiloaded viscoelastic nonlinear beams. Computers \& Structures, 46(2), pp. 215-218, 1993.

[7] Shirahatti, U.S \& Sinha, S.C., On the stability of perfect viscoelastic columns. Journal of Sound and Vibration, 174, pp. 57-68, 1994.

[8] Hewitt, J.S. \& Mazumdart, J., Buckling of viscoelastic plate. AIAA Journal, 15, pp. 451-452, 1977.

[9] Drozdov, A.D. \& Zhukhovitskii, D.M., Stability of circular plates from ageing viscoelastic material. Journal of Applied Mathematics and Mechanics, 50(2), pp. 208-212, 1986.

[10] Peng, F., Fu, Y. \& Liu, Y., On the durable critic load in creep buckling of viscoelastic laminated plates and circular cylindrical shells. Sci China Ser G-Phys Mech Astron, 51(7), pp. 873-882, 2008.

[11] Atanackovic, T.M., A modified Zener model of a viscoelastic body. Continuum Mech. Thermodyn, 14, pp. 137-148, 2002.

[12] Schmidt, A. \& Gaul, L., Finite element formulation of viscoelastic constitutive equations using fractional time derivatives. Nonlinear Dynamics, 29(1-4), pp. 37-55, 2002.

[13] Galucio, A.C., Deu, J.-F. \& Ohayon, R., Finite element formulation of viscoelastic sandwich beams using fractional derivative operators. Computational Mechanics, 33, pp. 282-291, 2004.

[14] Katsikadelis, J.T., Numerical solution of multi-term fractional differential equations. ZAMM Zeitschrift für Angewandte Mathematik und Mechanik, 89(7), pp. $593-608,2009$.

[15] Katsikadelis, J.T. \& Babouskos, N.G., Postbuckling analysis of viscoelastic plates with fractional derivative models. Engineering Analysis with Boundary Elements, 34, pp. 1038-1048, 2010.

[16] Katsikadelis, J.T., The analog equation method. A powerful BEM-based solution technique for solving linear and nonlinear engineering problems. Boundary Element Method XVI, ed. C.A. Brebbia, CMP, Southampton, pp. 167-182, 1994.

[17] Diethelm, K., The Analysis of Fractional Differential Equations, An Application-Oriented Exposition Using Differential Operators of Caputo Type, Springer-Verlag Berlin Heidelberg, 2010.

[18] Katsikadelis, J.T., Boundary Elements: Theory and Applications, Elsevier Science: London, 2002

[19] Katsikadelis, J.T. \& Armenakas, A.E., A new boundary equation solution to the plate problem. ASME Journal Applied Mechanics, 56, 364-374, 1989. 
46 Boundary Elements and Other Mesh Reduction Methods XXXIII

[20] Christensen, R.M., Theory of Viscoelasticity, Second Edition, Academic Press, New York, 1982.

[21] Mesquita, A.D. \& Coda, H.B., Boundary integral equation method for general viscoelastic analysis. International Journal of Solids and Structures, 39, pp. 2643-2664, 2002.

[22] Bulson, P.S., The stability of flat plates, Chatto \& Windus, London, 1970. 